\title{
Developing algorithms to enhance the sensitivity of cancellation tests of visuospatial neglect
}

\author{
NICK DONNELLY, RICHARD GUEST, and MIKE FAIRHURST \\ University of Kent, Canterbury, England \\ and \\ JONATHAN POTTER, ANTHONY DEIGHTON, and MAHOOL PATEL \\ Nunnery Fields Hospital, Canterbury, England
}

\begin{abstract}
We describe a set of algorithms that enhance the sensitivity of cancellation tests used in assessing visuospatial neglect. The algorithms can be readily implemented on a computer and can provide temporal and nontemporal data on strategies used during cancellation. We also present preliminary results from a group of 68 right-hemisphere brain-damaged patients and 12 age-matched control participants, which demonstrate the clinical significance of the measures we have defined.
\end{abstract}

Visuospatial neglect is a disorder in which stimuli appearing on the contralateral side to a brain lesion are ignored. Clinically, its presence is usually detected through a small number of simple tests, such as line bisection, copying, and freehand drawing. In line bisection, the task is to bisect a line in the center so that it is divided into equal halves. Copying and freehand drawing are selfexplanatory, with patients usually being required to produce geometrical forms or familiar, symmetrical objects, such as clocks. In each case, right-hemisphere braindamaged patients with visuospatial neglect ignore or omit critical information placed on the left-hand side of the page, with left-hemisphere patients producing the opposite result.

Although the condition has been reported since the late 19th century, it is only in recent years that it has become the subject of intense research interest (see Bradshaw \& Mattingley, 1995). Recent research has shown visuospatial neglect to be highly complex, resulting from damage to different neurological and cognitive systems. The neuroanatomical structure commonly associated with neglect is the inferior parietal cortex, although neglect is also found following damage to the dorsolateral frontal cortex and to subcortical structures, such as the thalamus and the basal ganglia. Theories based on neuropsychological case studies have suggested that cognitive deficits in attention, representation, or motor planning might underlie neglect. Therefore, in order to properly investigate neglect, it is necessary to develop assessment techniques capable

We acknowledge the contribution made by Dan Weeks and one anonymous reviewer for their excellent feedback and by the South East Thames Health Authority for funding the work. R.G. and M.F. are at the Electronic Engineering Labs at Kent. Correspondence concerning this article should be addressed to N. Donnelly, Department of Psychology, University of Southampton, Southampton SO17 1BJ, England (e-mail: nd5@soton.ac.uk). of simultaneously measuring multiple aspects of performance that are sensitive to different anatomical or cognitive systems.

In the present paper, we report a modified cancellation test in which patients are asked to cancel a number of target objects (e.g., Os, set among Xs). In our implementation of the cancellation task, cancellations are made on a sheet of paper placed on a graphic tablet connected to a PC. Each movement registered by the tablet is stored as a string of $x, y$ coordinates, and we describe how clinically relevant information concerning temporal and strategic aspects of performance can be extracted from these data.

\section{Implementing the $\mathrm{O} / \mathrm{X}$ Cancellation Task for Use With a Graphics Tablet}

Cancellation tasks have a long history in the study and detection of visuospatial neglect (Albert, 1973). They require that all the lines are crossed, all the Os (set among distractor Xs) have a line placed through them, or all of a set of small stars placed within a set of larger stars and words are crossed. Our version of the $\mathrm{O} / \mathrm{X}$ cancellation task consisted of an A4-sized $(29.7 \times 21 \mathrm{~cm})$ overlay, on which a series of $12 \mathrm{O}$ and $12 \mathrm{X}$ characters, or cancellation points, were printed (see Figure 1). Each character measured $7 \times 7 \mathrm{~mm}$, and the characters were arranged in a pseudo-random fashion. As in the standard task, the participants were required to cancel (cross out with a pen) all of the $O$ characters. Note that the pen marked the paper, in addition to transmitting coordinates to the computer.

\section{Data Capture}

The $\mathrm{O} / \mathrm{X}$ task was completed with an overlay positioned and fixed on a graphic digitization tablet (Wacom UD1212) connected to a PC. As the participants marked the overlays, the coordinates of a cordless pen were transmitted to the computer at a baud rate of $19,200 \mathrm{bits} / \mathrm{sec}$ ond. The spatial resolution of the tablet was 180 points $/ \mathrm{cm}$ 


$\begin{array}{cccccc}0 & & 0 & x & & 0 \\ 0 & x & x & & 0 & x \\ x & 0 & x & 0 & x & 0 \\ x & 0 & & x & 0 & x\end{array}$

Figure 1. $\mathrm{O} / \mathrm{X}$ cancellation task.

along both the $x$ and the $y$ axes. The spatial coordinate data, along with other pen position data (such as pressure and $x$ and $y$ tilt values, which we do not report here), were time-stamped with an offset from the start of data capture (in milliseconds) and stored in a separate ASCII data response file on the computer. The main benefits of this storage method (as opposed to real-time processing of the data) are twofold. First, complex feature extraction routines can be used on the data without the need to buffer the real-time data stream from the graphics tablet, and second, responses can be reanalyzed so that new features can be defined and extracted without the need for retesting.

\section{Analyses of Omissions}

To test for the number of omissions made by a participant, a mask file was matched against the data response file. The mask file was ASCII based and contained a list of target coordinates, an identification number, and a type (an $\mathrm{O}$ or an $\mathrm{X}$ ) for all of the targets on the cancellation task. To test whether a particular target had been canceled, target coordinates stored in the mask file were compared with coordinates in the data response file. This procedure mimics exactly the traditional measure of per- formance on a cancellation task. The closest target to a response coordinate was determined by the smallest Euclidean distance, defined by the formula

$$
D_{E}[(a, b),(c, d)]=\sqrt{(a-c)^{2}+(b-d)^{2}},
$$

where $(a, b)$ is the current response file coordinate and $(c, d)$ is the coordinate of a target contained in the mask file. If this distance was below a threshold of $5.6 \mathrm{~mm}$ ( $4 \mathrm{~mm}$ in each axis), the target point was listed as canceled.

\section{Analyses of Temporal Data}

Coordinates were stored every $50 \mathrm{msec}$ in the data response file. Simple measures, such as the overall (and mean) task execution time (time between the first and the last cancellation), were trivially easy to calculate. We also calculated many temporospatial parameters during the cancellation sequence, using intercancellation timings computed between successive pairs of points. Intercancellation times were split into movement, premovement, and drawing components. Movement time was defined as the time between the pen leaving its previous target and the start of the next cancellation, and premovement time as the duration for which the pen remained stationary after the completion of a cancellation before the next movement began. Drawing times were defined as the time from the beginning to the end of each cancellation.

For each of these measures, we computed the gradient of the linear trend (Press, Flannery, Teukolsky, \& Vetterling, 1992) of a graph showing time plotted against cancellation order. A positive gradient signified longer times toward the end of the sequence, whereas a negative gradient indicated reduced times between cancellations. Note that the first position within a sequence was ignored and was not included in subsequent calculations, since timings began when the first object was canceled.

Analyses of strategic data. The sequence of cancellation points from each participant was classified by matching against a series of archetypal patterns (stored in a third ASCII file, the sequence file) taken from

Table 1

O/X Task Sequence Definitions

\begin{tabular}{|c|c|c|c|c|c|c|c|c|c|c|c|c|c|c|c|c|}
\hline $\begin{array}{c}\text { Sequence } \\
\text { ID }\end{array}$ & $\begin{array}{c}\text { Traversal } \\
\text { Method }\end{array}$ & Start Side & $\begin{array}{l}\text { Start Position } \\
\text { in Column }\end{array}$ & $\begin{array}{l}\text { Traversal } \\
\text { Direction }\end{array}$ & & & & & & Sequ & tence & & & & & \\
\hline 1 & raster & left & top & vertical & 1 & 2 & 3 & 4 & 5 & 6 & 7 & 8 & 9 & 10 & 11 & 12 \\
\hline 2 & raster & left & bottom & vertical & 2 & 1 & 4 & 3 & 6 & 5 & 8 & 7 & 10 & 9 & 12 & 11 \\
\hline 3 & raster & right & top & vertical & 11 & 12 & 9 & 10 & 7 & 8 & 5 & 6 & 3 & 4 & 1 & 2 \\
\hline 4 & raster & right & bottom & vertical & 12 & 11 & 10 & 9 & 8 & 7 & 6 & 5 & 4 & 3 & 2 & 1 \\
\hline 5 & raster & left & top & horizontal & 1 & 5 & 11 & 2 & 9 & 3 & 7 & 12 & 6 & 8 & 4 & 10 \\
\hline 6 & raster & left & bottom & horizontal & 4 & 10 & 6 & 8 & 3 & 7 & 12 & 2 & 9 & 1 & 5 & 11 \\
\hline 7 & raster & right & top & horizontal & 11 & 5 & 1 & 9 & 2 & 12 & 7 & 3 & 8 & 6 & 10 & 4 \\
\hline 8 & ister & right & bottom & horizontal & 10 & 4 & 8 & 6 & 12 & 7 & 3 & 9 & 2 & 11 & 5 & 1 \\
\hline 9 & snake & left & top & vertical & 1 & 2 & 4 & 3 & 5 & 6 & 8 & 7 & 9 & 10 & 12 & 11 \\
\hline 10 & lake & left & bottom & vertical & 2 & 1 & 3 & 4 & 6 & 5 & 7 & 8 & 10 & 9 & 11 & 12 \\
\hline 11 & lake & right & top & vertical & 11 & 12 & 10 & 9 & 7 & 8 & 6 & 5 & 3 & 4 & 2 & l \\
\hline 12 & snake & right & bottom & vertical & 12 & 11 & 9 & 10 & 8 & 7 & 5 & 6 & 4 & 3 & 1 & 2 \\
\hline 13 & snake & left & top & horizontal & 1 & 5 & 11 & 9 & 2 & 3 & 7 & 12 & 8 & 6 & 4 & 10 \\
\hline 14 & snake & left & bottom & horizontal & 4 & 10 & 8 & 6 & 3 & 7 & 12 & 9 & 2 & 1 & 5 & 11 \\
\hline 15 & snake & right & top & horizontal & 11 & 5 & I & 2 & 9 & 12 & 7 & 3 & 6 & 8 & 10 & 4 \\
\hline 16 & snake & right & bottom & horizontal & 10 & 4 & 6 & 8 & 12 & 7 & 3 & 2 & 9 & 11 & 5 & 1 \\
\hline
\end{tabular}


studying the performance of normal participants (as is shown in Table 1). These patterns were formed by using assigned identification numbers for each cancellation location (Figure 2) and two defined sets of traversal method, categorized as either a raster or a snake. A correctness score was calculated between each of the model sequences and the actual sequence, using a goodness-offit measure (the location identifier in position $n$ being identical to that in the model sequence). Because of the possibility of participants using a normal strategy but not beginning in the leftmost or rightmost location, the test sequences were recursively barrel-shifted one position to the right, with the sequences "wrapping around" at the limits of the display. After each barrel shift, a goodnessof-fit score was calculated, and the procedure was repeated until the sequence returned to its original position (see Figure 3). The highest goodness-of-fit score was used to classify the sequence. Computing the highest number of matches in this way provided three other pieces of information: the number of barrel shifts required to obtain the highest match, the predefined sequence that produced the highest match, and the start point for cancellations. Only O characters were included in the assessment of sequences. Before classification, the test attempt sequence was preprocessed in order to remove all X cancellations, since we were only interested in correct cancellation locations in sequences. Duplications were also removed, so that a direct comparison could be made against the reference patterns.

The number of path crossings (intersections) within a completion sequence was also computed (see Figure 4 for an example of a strategy with a single crossing point). Calculating intersections required using the sequence file with reference to the mask file to obtain target coordinates. Consecutive pairs of cancellation points within the sequence formed virtual lines indicating the sequence path. Each virtual line was compared against others in the sequence, to test for crossings using a fast line intersection routine (the case in which one line starts and another ends at the same point-i.e., the next line in sequence-was ignored). Line crossings were detected by the following method: The two line segments for inspection were defined by the coordinates of end points: Line 1 from $\left(x_{1}, y_{1}\right)$ to $\left(x_{2}, y_{2}\right)$ and Line 2 from $\left(x_{3}, y_{3}\right)$ to $\left(x_{4}, y_{4}\right)$. The standard equation for a straight line $0=a x+b y+c$ was used to define each segment, giving

$$
0=a_{1} x+b_{1} y+c_{1} \text { for Line 1(Equation 1) }
$$

and

$$
0=a_{2} x+b_{2} y+c_{2} \text { for Line } 2 \text { (Equation 2), }
$$

where

$$
\begin{aligned}
& a_{1}=\left(y_{2}-y_{1}\right), b_{1}=\left(x_{1}-x_{2}\right) \text { and } \\
& c_{1}=\left(x_{2} * y_{1}\right)-\left(x_{1} * y_{2}\right)
\end{aligned}
$$

and

$$
\begin{aligned}
& a_{2}=\left(y_{4}-y_{3}\right), b_{2}=\left(x_{3}-x_{4}\right) \text { and } \\
& c_{2}=\left(x_{4} * y_{3}\right)-\left(x_{3} * y_{4}\right) .
\end{aligned}
$$

A simple check, to see whether the segments intersected, was performed by substituting sets of end points of Line 2 into Equation 1. If the result of both equations were of the same sign, both end points of Line 2 were above (both negative) or below (both positive), and the lines did not intersect. A 0 result from the equation signified that the end point lies on Line 1 . For verification, the same procedure was repeated for Line 1, substituted into Equation 2. Points of intersection $\left(x_{i}, y_{i}\right)$ were defined as

$$
y_{i}=\frac{-\left(a_{1} x_{i}+c_{1}\right)}{b_{1}}=\frac{-\left(a_{2} x_{i}+c_{2}\right)}{b_{2}},
$$

which gives

$$
x_{i}=\frac{-c_{1} b_{2}+c_{2} b_{1}}{-a_{2} b_{1}+a_{1} b_{2}}
$$

and

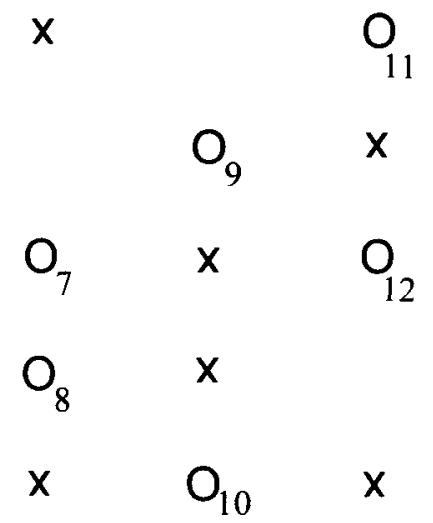

Figure 2. Location identification numbers. 

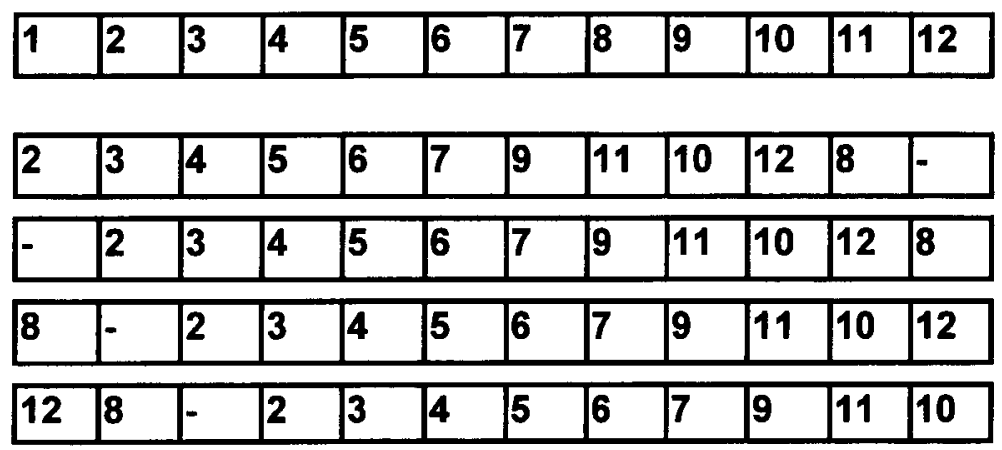

Model sequence

Actual sequence (0)

Right shift 1 (6)

Right shift 2 (2)

Right shift 3 (1)

Figure 3. Barrel shift correctness of match operation. The figures in parentheses indicate the correctnessof-score match.

$$
y_{i}=\frac{-\left(a_{1} x_{i}+c_{1}\right)}{b_{1}} .
$$

\section{Preliminary Data Case Study}

The measures that we have outlined above need to be shown to have some theoretical or clinical significance, to make it worthwhile to extract them. We now present a preliminary report of a group of 68 right-hemisphere patients and 12 age-matched controls. Our primary aim in presenting these data is to show that the measures we have defined can discriminate between brain-damaged patients with visuospatial neglect, brain-damaged patients without visuospatial neglect, and age-matched participants.

All the brain-damaged patients were administered the Rivermead Behavioural Inattention Test (BIT; Wilson, Cockburn, \& Halligan, 1987) in order to classify them into a brain damage with visuospatial neglect group or a control group. Twenty-eight (42\%) patients were classified as having visuospatial neglect. Here, we summarize the results from the omission, temporal, and strategic measures for visuospatial neglect, brain-damaged, and age-matched control groups.
Omissions. Only one control made any omissions, and this participant scored $11 / 12$. Only $2 / 40$ brain-damaged control patients made omission errors. This compares with the brain-damaged neglect group, where 14/28 omitted canceling at least one of the targets. This rate of agreement with the BIT is expected because of differences in test sensitivity. Note that the BIT does not include an OX cancellation test.

Timing. The overall task execution time varied between 7.6 and $24.8 \mathrm{sec}$ for age-matched controls, 9.1 and $50.1 \mathrm{sec}$ for brain-damaged control patients, and 3.4 and $66.4 \mathrm{sec}$ for those brain-damaged patients with visuospatial neglect. Three visuospatial neglect patients were either below or above the range of both control groups. In the case of two patients, they canceled so few items ( 2 and 4 , respectively) that the short execution times simply reflect this fact. However, one patient canceled 10 items but took longer than any of the control participants to do it.

The most interesting data come from studying movement and premovement times. Analysis of individual slopes for age-matched and brain-damaged control participants provided limits for comparison with the neglect population. In $8 / 28$ cases (see Table 2 ), patients with

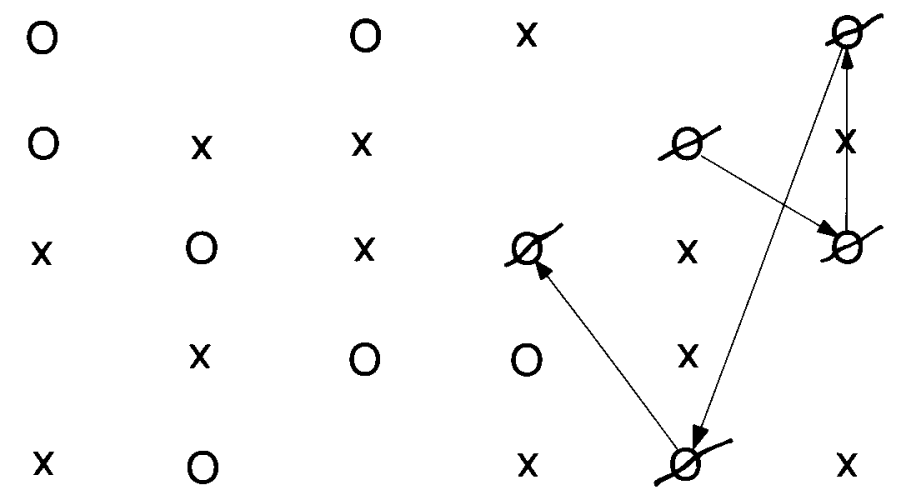

Figure 4. Path with single crossing point. 
Table 2

Distribution of Abnormal Premovement and Movement Gradients for Each Visuospatial Neglect Patient, With Lesion Locus Specified

\begin{tabular}{lcc}
\multicolumn{1}{c}{ Lesion } & Premovement & Movement \\
\hline Internal capsule & - & - \\
Frontoparietal & - & - \\
Occipital & - & - \\
Parietal & - & + \\
Parietal & - & - \\
Periventricular & + & - \\
Parietal & - & - \\
Occipitothalamic & - & - \\
Internal capsule & - & - \\
Temporoparietal & + & - \\
Internal capsule & - & - \\
Frontal & - & - \\
Parietal & - & - \\
Temporoparietal & - & - \\
Frontoparietooccipital & + & + \\
Parietal & - & - \\
Pontine & - & - \\
Periventricular & - & - \\
Parietal & + & + \\
Temporooccipitobasal ganglia & + & - \\
Temporoparietal & - & - \\
Parietal & - & - \\
Occipital & - & - \\
Parietal & - & - \\
Basal ganglia & - & - \\
Temporoparietal & - & - \\
Parietal & - & - \\
Cerebellum & - & - \\
\hline
\end{tabular}

Note--+ indicates abnormal gradient; - indicates gradient within limits of age-matched and brain-damaged controls. The first 14 patients listed made omission errors.

visuospatial neglect produced slope values outside of these limits. Of these cases, $5 / 8$ were patients who had made no omission errors. Adding these patients to those who made omissions means that we have increased our rate of agreement with the BIT to $19 / 28(68 \%)$. None of the visuospatial neglect patients fell outside of the limits provided by age-matched and brain-damaged controls for drawing time.

Sequencing. The completion rates (as a function of the number of items canceled) that can be accounted for by any one of the stored strategies was $100 \%$ for agematched and brain-damaged with neglect patients and $88 \%$ for brain-damaged control participants. The modal response strategy adopted by age-matched controls was strategy $1(46 \%)$; by brain-damaged patients without neglect, it was strategy $1(33 \%)$, followed by $11(10 \%)$; and by brain-damaged patients with visuospatial neglect, it was strategy $3(21 \%)$, followed by strategies 12 and 9 (both $18 \%$ ). These differences in strategy largely reflect mirror image strategies, with $92 \%$ of the age-matched controls starting cancellations on the left side of the page, as compared with $68 \%$ of the right-brain-damaged patients without neglect and only $25 \%$ of the right-braindamaged patients with visuospatial neglect. By adding those patients who started on the right side to our list of neglect patients making omissions and outside normal limits of premovement and movement timing, we find that $25 / 28(89 \%)$ of the patients originally defined as having visuospatial neglect from the BIT were also detected in the present study. Note that the presence of patients starting on the right in the brain-damaged control group means that this measure is likely to indicate visuospatial neglect but, in a proportion of cases, might simply indicate the presence of brain damage.

There were no intersections found in the age-matched control population, but they ranged between 0 and 14 for the brain-damaged patients without visuospatial neglect and were found in $12 / 40$ cases. Intersections ranged from 0 to 5 for brain-damaged patients with visuospatial neglect and were found in $8 / 28$ cases. The presence of intersections in both brain-damaged groups shows that this measure only indicates the presence of brain damage and is not specific to visuospatial neglect.

These preliminary data reveal that our implementation of the cancellation tasks is sensitive to visuospatial neglect. However, in addition to measuring omissions, they reveal that a subset of patients with visuospatial neglect do not maintain the same pattern of movements and premovements as do age-matched or brain-damaged controls and that they have a strong tendency to begin canceling on the right of the page. By using these three measures, 25/28 of the patients originally identified by the BIT as having visuospatial neglect were also detected by the present system from a single $\mathrm{O} / \mathrm{X}$ cancellation test.

\section{Conclusion}

Implementing the $\mathrm{O} / \mathrm{X}$ cancellation task on the graphics tablet has allowed visuospatial neglect to be automatically identified by the traditional measure of counting the number of omissions made by patients, testing for distinctive patterns of premovement and movement timings and for patients' starting positions. The automation of scoring omissions is relatively trivial and would not, by itself, advance the detection or study of the condition. However, through developing and implementing a set of algorithms, we have shown how other important and clinically relevant information can be elicited from such a simple test. In particular, in this report, we have focused on temporal (measures of movement and premovement) and strategic (start points and intersections) factors during cancellation.

We noted earlier how visuospatial neglect results from damage to different neurological and cognitive systems. It is possible that the fine-grained measures we have described might be able to distinguish between them. From our preliminary case study, only in the case of damage to the parietal cortex, rather than to other structures, are there sufficient numbers to make reasonable comparisons. If damage includes the parietal cortex, there is a greater probability of some disorder of premovement or movement than if the damage does not include the parietal cortex (.4 vs. .15). Given the relative specificity of disorders of premovement and movement to the parietal cortex, the probability of such disorders can be compared with the probability of making omissions following dam- 
age that includes the parietal cortex $(.47)$ versus other types of brain damage (.54). Although occurring less frequently, disorders of premovement and movement are more likely to indicate parietal damage than are omissions.

Further research will focus on enhancing the measures we have identified as relevant and on exploring their validity on more complex cancellation tasks. It is also likely that future research will show another advantage of implementing the $\mathrm{O} / \mathrm{X}$ cancellation task via the graphics tablet and computer. As yet, it is impossible to directly compare patients across time, to allow direct comparison of performance after spontaneous recovery or treatment regime, using any other measure than number of omissions. We believe that important new data will be gained by studying the strategic and temporal aspects of performance at different stages of recovery. Only the system that we have described affords this opportunity.

\section{Availability}

The software routines are available from R. Guest, Department of Electronics, University of Kent, Canterbury, England.

\section{REFERENCES}

AlBERT, M. L. (1973). A simple test of neglect. Neurology, 23, 658-664. Bradshaw, J. L., \& Mattingley, J. B. (1995). Clinical neuropsychology. San Diego: Academic Press.

Press, W. H., Flannery, B. P., Teukolsky, S. A., \& Vetterling, W. T. (1992). Numerical recipes in $C:$ The art of scientific computing. Cambridge: Cambridge University Press.

Wilson, B., Cockburn, J., \& Halligan, P. W. (1987). Behavioural inattention test. Titchfield, Hants: Thames Valley Test Company.

(Manuscript received September 21, 1998 . revision accepted for publication January 28, 1999.) 